\title{
References
}

Barker, S. 2009. Dispositional monism, relational constitution and quiddities. Analysis 69: 242-50.

Bird, A. 2007. Nature's Metaphysics: Laws and Properties. Oxford: Clarendon Press.

Dipert, R. 1997. The mathematical structure of the world: the world as graph. The Journal of Philosophy 94: 329-58.

Erdös, P. and A. Rényi. 1963. Asymmetric graphs. Acta Mathematica Academiae Scientiarum Hungaricae 14: 295-315.

Harary, F. 1969. Graph Theory. Reading, MA: Addison-Wesley Publishing Company.

Lowe, E.J. 2006. The Four-Category Ontology: A Metaphysical Foundation for Natural Science. Oxford: Clarendon Press.

Oderberg, D.S. 2011 (in press). No potency without actuality: why graph theory cannot rescue a world of pure powers from the regress/circularity objection. In Contemporary Aristotelian Metaphysics, ed. T. Tahko. Cambridge: Cambridge University Press.

Robinson, H. 1982. Matter and Sense. Cambridge: Cambridge University Press.

Wilson, R.J. 1996. Introduction to Graph Theory, 4th edn. Harlow: Addison Wesley Longman Limited.

\section{The world as a graph: defending metaphysical graphical structuralism}

\author{
Nicholas SHackel
}

Metaphysical graphical structuralism is the view that at some fundamental level the world is a mathematical graph of nodes and edges. Randall Dipert has advanced a graphical structuralist theory of fundamental particulars and Alexander Bird has advanced a graphical structuralist theory of fundamental properties. David Oderberg has posed a powerful challenge to graphical structuralism: that it entails the absurd inexistence of the world or the absurd cessation of all change. In this article, I defend graphical structuralism. A sharper formulation, some theorems about such structures, and careful attention to the interaction of metaphysical and mathematical features, shows that the absurdities depend on assumptions that are not essential to the view and brings to light a surprising fact about the necessary structure of fundamental properties.

Dipert (1997) proposes that 'the concrete world is a single, large structure induced by a single, two place, symmetric relation... [which is] best analyzed as a certain sort of graph' (1997: 329), namely a graph of nodes and edges, 
where nodes are the relata of the fundamental relation and edges link nodes that stand in that relation. This is a monism because the nodes and edges have no distinctness on their own, but rather, have identity only through their location in the world structure and this amounts to their identity depending on the entire world. Hence the proper parts of the world, which are all the proper subgraphs of the world, have no independent identity, whence, strictly speaking, there is just one thing that is - the whole world.

In his original paper, Dipert vacillates between taking the graph as a description of the world and taking the graph to be the world. This introduces some unnecessary obscurities. For example, in the quotation just given the world is supposed to be both a single large structure and yet also metaphysically induced by a relation, but if the latter then it is induced on some things metaphysically prior to it by an equally metaphysically prior relation. This is not strictly compatible with the monism that he clearly intends, since the plurality of things and relation is metaphysically prior to the world whereas monism requires it to be the other way around. The vacillation also causes some unnecessary obstacles in defending a structural criterion of identity for nodes.

These difficulties can be dispensed with by formulating the structuralist position strictly in terms of the identity of graph and world. The world is a complex unity whose parts are metaphysically dependent on the whole and whose finest level of dependent entities are nodes and edges; hence the world is a graph, whose structure gives rise to an internal twoplace symmetric relation that stands between any pair of nodes linked by an edge. ${ }^{1}$

David Oderberg's (2011: 6-9) challenge to particularist graphical structuralism runs, in brief:

(1) Suppose the world is a graph.

(2) If it is a graph it is an asymmetric graph.

(3) Any asymmetric graph can be turned into symmetric graph by the removal of edges.

(4) The loss of less than all the edges of the world (specifically, the loss of all those not part of some symmetric subgraph, a loss consequent on certain nodes going out of existence) metaphysically entails the non-existence of the entire world. $(1,2,3)$

(5) Therefore, the world is not a graph. (1, 4 reductio ad absurdum)

The second premiss is a consequence of the world being a graph in which the nodes of the world have identity. Because Dipert is defending a monism

1 I shall ignore infinite graphs which introduce complications without adding anything essential to our discussion. 
he wants a purely structuralist criterion of identity: 'entities are individuated ... solely by their graph-theoretic structural features' (1997: 329). ${ }^{2}$

The fourth line can be resisted. It doesn't immediately follow that, for some symmetric subgraph of the world, if we removed edges that distinguish the asymmetric graph from that subgraph, the entire world goes out of existence. What the structuralist's criterion of identity entails is that nodes which were previously distinct would become identical, in a way determined by the symmetry of the symmetric subgraph. I think the manner is clear enough if I say that the world would collapse to the 'nearest' asymmetrical graph - by which I mean the asymmetrical graph you get from the symmetrical subgraph after identifying nodes with the same kind of location.

Now of course, this is uncomfortable for the structuralist, indeed a mass of further peculiarities might emerge depending on the precise nature of the original graph. Changes would be abrupt, contradicting principles of continuity, and peculiarly arbitrary, in the sense that distinct entities originally far apart ${ }^{3}$ might suddenly become one. The graph might become disconnected, so one world might shatter into many. If the order of the symmetry group is greater than two then there may be more than one nearest asymmetrical graph, depending on how distance is to be measured, ${ }^{4}$ leaving it indeterminate which asymmetric graph supersedes. ${ }^{5}$

All of this is, I think, grist to Oderberg's mill, and yet still not as devastating for the structuralist as the claimed implication of going out of existence. The argument to inexistence might be saved given further argument showing that agreed metaphysical principles entail certain additional graph theoretical properties to be necessary for the world graph, since any such properties are likely to be disrupted by collapses, and might not be preserved, thereby entailing inexistence rather than collapse to a smaller world. This, however, would take more detailed argumentation.

So the route to inexistence can be blocked. Removal of edges may lead only to the collapse to a smaller asymmetric graph due to the merging of previously distinct nodes. From this I think it is evident that the fourth line (and hence Oderberg's argument) is implicitly relying on the necessity of identity,

2 Let: the kind of a node be the number of edges incident to it; an adjacency property of a node be a property of the form 'being a node of kind $k$ adjacent to a node of kind $m$ which is adjacent to a node of kind $n .$. which is adjacent to a node of kind $j$ '; the kind of location of a node be the complete set of adjacency properties of that node; let a node be graphically unique iff there is no other node in the graph that has the same kind of location. Then graphical uniqueness is a necessary and sufficient criterion of identity. Premiss 2 follows quickly from all nodes being graphically unique.

3 Distance between two nodes = shortest path linking them.

4 A definition of distance may be available from features of the symmetry group of the symmetric graph, or perhaps from its quotient groups.

5 This last might, I suppose, be considered a kind of metaphysical explanation for how there could be ontic randomness in the world. 
since that would rule out any such merging, leaving inexistence as the only alternative.

Presupposing necessity of identity is not dialectically unreasonable. Nevertheless, it is not clear that the structuralist must take the necessity of identity as a constraint on his theory, most especially when we are speaking of the identity of the dependent entities within a monist metaphysic. He might rather think that whether identity is necessary or contingent should follow from his structural criterion of identity.

I turn now to the question of absurdity in the fourth line. Oderberg thinks Dipert faces a dilemma: 'either... all or some individuals exist necessarily... [or] the world can be destroyed by the removal of some individuals' (2011: 6-7). He rejects the first horn since 'surely all individuals in the physical world exist contingently' hence only the absurdity of world destruction remains.

It is worth noting for a start that Dipert is explicitly necessitarian:

There is one and only one asymmetric graph that...constitutes the world and thus only one alethically 'possible' world or graph. (1997: 356 n. 45)

On this account it's impossible for the world graph to be other than it is and hence any change at all entails non-existence. So here the fourth line is true and yet there is no absurdity in the loss of even a single edge entailing non-existence. The absurdity would have to be in the necessitarianism.

What about the contingency of physical individuals? A natural way to understand Dipert's 'sketch of how structuralism could analyze... [the physical world]' (1997:355) is to take it to be committed to four-dimensionalism about time and space and perdurantism about persistence:

there are... regular connecting paths among a certain subset of objects (subgraphs) that we conceive as mid-sized, phenomenal 'physical objects,' and that these paths have the structure we identify as space and time. (Dipert 1997: 356 n. 45)

So a physical individual is a collection of subgraphs with certain similarities and joined in a certain way, each of which subgraphs is a temporal part of the individual. I think it is clear that this allows physical individuals to come into and go out of existence and hence the necessity of the existence of the nodes does not entail the necessity of physical individuals. Thus Dipert's necessitarianism is compatible with individuals of the physical world being contingent. ${ }^{6}$ 
What about the fundamental physical individuals of physics? Here I think that Oderberg's central remark is potentially misleading:

So, in the physical case, say Dipert's model of wholly relational individuals, were any to go out of existence so would their relations to the remaining individuals to which they were previously related. (2011:6)

The plausibility of taking the nodes of the world graph to be contingent, and hence capable of going out of existence, comes from taking the nodes of the world graph to be the fundamental physical individuals of physics, a plausibility which Oderberg has set up by earlier calling them 'qualitatively identical micro-particles' (2011: 5). But Dipert explicitly rejects this when he says:

Physical objects, even the finest subatomic particles, certainly do not correspond to vertices. Instead, they themselves are composite entities, subgraphs of the world graph. Physical microstructure is graphtheoretic macrostructure. (1997: 356)

In this metaphysic the nodes and edges of the world are necessary existents, but only in the same sense that in a standard four-dimensionalism, temporal parts are necessary existents. Yes, if the nodes and edges are physical individuals then some physical individuals are necessary. But the contingency of what we usually call physical individuals, including the fundamental physical individuals of physics, remains.

The apparent absurdity of the loss of some edges resulting in the inexistence of the world depended on taking the nodes and edges to be contingent individuals of familiar physical varieties. But in fact, the contingency of the latter individuals is compatible with the necessity of nodes and edges and the necessity of the latter defeats the absurdity of their loss resulting in inexistence.

If there is an absurdity remaining it is in Dipert's necessitarianism. Yet, we can now see that the defeat of the absurdity does not depend on necessitarianism. We can allow that there are many possible worlds, each an asymmetric graph. ${ }^{7}$ Neither the actual world nor any possible world is up for having some of its edges deleted at some point in time since the existence of the nodes and edges of a world are metaphysically entailed by the existence of the world. So it is impossible to get rid of the nodes and edges of a world and the argument to the absurd inexistence is blocked.

Bird (2007) develops a dispositional essentialist theory of properties, in which there is a fundamental level of properties, which properties are potencies. The fundamental level is fundamental because it is the 'non-redundant basis of causal relations' and includes the 'minimal base on which all else

7 So now we have contingent individuals in the sense that they might never have existed. 
supervenes' (2007: 14). From here on, we speak only of properties of the fundamental level.

An objection to Bird's theory is that if all properties are potencies they have nothing intrinsic to fix their identity, only their stimulus and manifestation conditions, which in turn are further potencies. Hence their identity depends on their relation to other potencies, and this leads to a vicious regress or circularity, leaving their identity indeterminate. Bird's defence to this objection is that the potencies are the nodes of an asymmetric directed graph in which the directed edges (arrows) join a potency to the potency that is its manifestation condition. ${ }^{8}$ Identity is secured by the graphical uniqueness of each node.

Oderberg's challenge to property graphical structuralism 'applies... to Bird's graph theoretical structure of pure potencies at the fundamental level'. His first argument is that Bird cannot remain neutral between Aristotelean immanence and Platonic transcendence for properties but is committed to transcendence. The basis for this is that under Aristotelean immanence 'by destroying every instance of a universal I destroy the universal' and hence by destroying 'every instance of enough potencies so that the remaining potencies constituted a symmetric graph' (2011: 7) the world would go out of existence. ${ }^{9}$

This argument does not succeed on Armstrong's version of immanence:

We certainly should not demand that every universal should be instantiated now... The principle of instantiation should be interpreted as ranging over all time. (Armstrong 2008: 65)

Immanent properties exist provided there is some time at which they are instantiated. That there is a time after which some of them are no longer instantiated doesn't amount to their inexistence. Bird's dispositional essentialism does not rule out 'any time' immanence of potencies.

There are two ways to understand Oderberg's premiss of destroying the instances to destroy the universal. He might mean that he destroys all instances over all times so the universal never exists. This, however, is just a covert way of contrasting possible worlds, not a way of specifying an event in a world that leads to its inexistence. So he can only mean that at a certain time he destroys all the instances and after that time the universal is no longer

8 For completeness, Bird takes it that we need a graph with two types of directed edges, one for manifestation conditions and the other for stimulus conditions. If two types are needed then asymmetry is even easier so confining ourselves to standard directed graphs, as we shall do for brevity, doesn't help Bird.

9 When Oderberg says 'we'd better hope that there are not a mere six potencies, since the loss of any one of them would destroy all the rest...' (2011:7) he has slipped up. This would be true were Bird's graph a simple graph rather than a directed graph, since there are no asymmetric simple graphs with between 2 and 5 nodes. However, the smallest potency graphs have 3 nodes (Lemma 2, see Appendix). 
instantiated. But that doesn't entail inexistence of the world under 'any time' immanence of potencies, since that doesn't amount to destroying the universal. He needs the additional premiss that the only properties that exist are the properties that are instantiated now, and it is hard to see how to motivate that premiss except by taking presentism as a premiss.

At root, Oderberg is appealing to some kind of very general conservation principle, something like that you can't get absolutely nothing out of something. The absurdity is not simply in how few potencies lost suffice for inexistence but rather, in there being anything at all that could occur in the natural succession of worldly events that would result in the sudden and complete inexistence of the world. Whilst this may 'strike us as bizarre' (2011: 7), I'm not sure that we know it to be absurd. Supposing that it is impossible, the question is whether, under presentism, immanence and dispositional essentialism, there might be a natural succession of worldly events, possibly immensely complex, which would result in just the right potencies no longer being instantiated, leaving only a symmetric graph of potencies and hence leading to the inexistence of the world. The answer to that question is more likely to be yes if losing only one potency suffices for inexistence. Nevertheless, it might depend on the actual graph of potencies. Furthermore, impossibility of the world's inexistence in that way might rather be a constraint on possible potency graphs (and hence on possible worlds) than a refutation of dispositional essentialism. Or perhaps its impossibility means that it is impossible to destroy all the instances of the right potencies. Against this, however, consider the situation where all but the last instance of the last right potency, say a photon energy level dependent for its instantiation on diffraction by the wing of a particular butterfly, have been destroyed. It isn't credible that that butterfly is now metaphysically impossible to destroy. So Bird's dispositional essentialism might well be incompatible with the conjunction of immanence and presentism.

Oderberg then runs an argument against Bird's potencies understood as Platonic transcendent properties. The objection here is not that the loss of potencies leads to inexistence, because being Platonic they cannot be lost. Rather, it is that if a single potency was not instantiated at all, 'no power could manifest itself - and so the fundamental level would grind to a halt', a consequence of which would be 'the entire universe's grinding to a halt'10 (2011: 8).

What Oderberg means here by the fundamental level is unclear. On the one hand, talk of it grinding to a halt implies that it is usually undergoing change. That sounds like he means a fundamental level of particulars. On the other hand, in the context, he ought to mean Bird's fundamental level, but that is incompatible with talk of it grinding to a halt, because it is the changeless transcendent graph of potencies. I take it that the absurdity aimed at is that

10 Since everything supervenes on the fundamental level. 
all change will cease. Here is my reconstruction of what I take to be the argument for that conclusion: ${ }^{11}$

(1) A potency essentially requires a manifestation condition.

(2) Under dispositional essentialism a manifestation condition is a potency.

(3) Suppose a single potency, $P$, was instantiated nowhere and consider all those potencies, $Q$, for which it is the manifestation condition.

(4) Dependency of Manifestation: For each instantiation of each of those potencies the potency instantiated, $Q_{i}$, would not be able to manifest its manifestation condition, $P$, just because $P$ is unavailable due to being nowhere instantiated.

(5) Regress: Furthermore, each potency, $Q_{i}$, is in turn the manifestation condition for yet further potencies, $R$, each of which will now, due to $Q_{i}$ being unable to manifest $P$, be unable to manifest $Q_{i}$.

(6) The regress continues and will eventually reach all potencies. ${ }^{12}$

(7) Hence no potency will be able to manifest.

(8) Change is, or is the result of, the manifestation by potencies of their manifestation condition.

(9) Therefore, all change will cease.

Premisses 1, 2 and 8 are agreed and the supposition of premiss 3 seems possible.

Line 4 looks vulnerable to the objection that the occurrence of the stimulus condition of $Q_{i}$ could lead to $Q_{i}$ bringing about a new instantiation of $P$. For example, suppose no squark is spinning and up squarks are disposed to spin on bumping into down squarks. ${ }^{13}$ Why shouldn't a bump bring about a new instantiation of spinning? New instantiations of properties in this manner are entirely normal.

Oderberg would say the problem is that 'every time a stimulus is applied, whatever produces its manifestation miraculously pops back into existence', or is guaranteed to pop back into existence, or is guaranteed to exist (2011: 8 ). What produces the manifestation is the stimulus potency of the potency that manifests, and that never went out of existence. So Oderberg has to be speaking not of the producer of the manifestation but its bearer.

11 Lines 3, 4, 5 and 6 are what I make of 'The necessarily existing powers would all still be relationally defined... but none of their tokens would be able to manifest themselves if all of the tokens of a single power type ceased to exist. For the tokens whose type was essentially related to this single power type would themselves not be able to manifest themselves, nor would the tokens whose type was essentially related to this second type, and so on.' (2011: 8)

12 It can only terminate at potencies that lack incoming arrows.

13 So spinning is the manifestation condition of up-ness, whose stimulus condition is down-ness. 
What Oderberg intends is that $P$ is instantiated nowhere because only a certain kind of particular can instantiate it and no particulars of that kind exist. So the reason $Q_{i}$ cannot manifest in a new instantiation of $P$ is because no particulars that can instantiate $P$ exist and it would require a miracle or an arbitrary guarantee for them to appear. The problem with this is twofold. First, quantum physics seems to be full of particles popping into existence. Second, the necessitarian aspect of dispositional essentialism makes the theory seem well equipped to underwrite a guarantee. Potencies necessitate their manifestation conditions when stimulated and hence the existence of a bearer is necessitated. This sounds surprising but consider this example. Suppose the raised energy level, $\delta E$, instantiated by a particular electron encounters its stimulus condition. The electron collapses to its lower energy level and emits a previously non-existent photon having frequency $\nu=\delta E / h$, which last is the manifestation condition of $\delta E$. Now suppose just prior to this event no photons exist because they have all been absorbed by electrons. Oderberg's defence of Line 4 requires the non-existence of all the other photons to block the photon emission, an emission which would have occurred if just one other photon existed. This is not credible.

Line 5 seems to assume that for a potency, $R_{i}$, to manifest its manifestation condition $Q_{i}$ requires that its manifestation condition $Q_{i}$ manifest its own manifestation condition, $P$. But that assumption is false. For $R_{i}$, to manifest its manifestation condition, $Q_{i}$, requires only that $Q_{i}$ instantiate quiescently, not that $Q_{i}$ also manifest.

There is an inclination to reject the possibility of quiescent instantiation because some chains of potencies result in the manifestation of one power requiring not simply the instantiation but also the manifestation of the power that is its manifestation condition. For example, an electron (belonging to an atom) has the power, $D$, to absorb photons, and $D$ itself manifests by its manifestation condition, $\delta E$, an increased energy level of the electron. A photon has the power, $C$, to be absorbed, and $C$ manifests itself by photon absorption, that is to say, by its manifestation condition, $D$, not merely instantiating, but in addition, manifesting itself in its manifestation condition, $\delta E$. So here we had a chain where manifestation of $C$ could not stop at the instantiation of manifestation condition, $D$, but had to continue. However, it is clear that the chain can stop at the instantiation of $\delta E$, at the increased energy level, and so $\delta E$ instantiates quiescently. So it seems perfectly possible that one potency should instantiate quiescently and for it to manifest to require stimulation by a different potency.

When $Q_{i}$ is the manifestation condition of $R_{i}$ and $R_{i}$ is the stimulus condition of $Q_{i}, R_{i}$ both manifests $Q_{i}$ and stimulates $Q_{i}$ thereby requiring $Q_{i}$ to manifest in turn. As we just saw, this can be true for some potencies but Line 5 needs it to be true for all potencies. If that were the case stimulus and manifestation would have lost their distinction (since they, too, are identified structurally). Alternatively, if quiescent instantiation of potencies is ruled out 
and instantiation is manifestation then the manifestation relation and the stimulus relation are really one and the same relation regarded from different ends of the arrow. That seems doubtful. ${ }^{14}$

Oderberg clearly thinks it obvious that the regress reaches all potencies, giving it no more than 'and so on' in his argument. In so doing he has missed a number of difficulties. Provided Line 5 is true then the regress will continue backward to some extent. But what is the guarantee that, as Line 6 puts it, the regress reaches all potencies, and does so irrespective of which potency is uninstantiated? Investigating whether that is true is non-trivial. Although there are potency graphs for which the regress will reach all potencies (Theorem 1, see Appendix), there are also potency graphs for which the regress will not reach all potencies (Theorem 2) and that the regress can leave untouched a subgraph of the original potency graph that is itself a potency graph (Theorem 3). In the last case at least, only some processes of change would cease; others would remain and change itself would not cease. However, if the potencies must each have only one manifestation condition, a plausible constraint at the fundamental level, and if the potency graphs must be connected, then a potency graph must be a 'snowflake' (Snowflake Lemma) and as a consequence of having that structure the regress can reach all potencies provided it starts from the right node (Uniquely Manifesting Potencies Regress Theorem).

So this argument is by no means conclusive. Both of the crucial lines, Dependency of Manifestation and Regress, face serious difficulties. If those difficulties are resolved, its success still depends on the actual graph of potencies allowing the regress to reach all potencies. But even then, an obstacle remains. To get rid of all instantiations of a potency requires removing an entire class of entities of a fundamental natural kind. It is not clear that it is absurd that there should be one such class whose removal results in the cessation of all change. For example, in a simple universe with only the electromagnetic and the nuclear forces of the standard model of particle physics, removing all the bosons would be removing all the force carrying particles. It does not seem so absurd that if you remove all the force carrying particles all change would cease. But if it is not absurd then the argument cannot be wielded as a reductio of Platonic dispositional essentialism.

In conclusion, then, Oderberg has posed a serious challenge to some metaphysical theories committed to graphical structuralism. We have seen that graphical structuralism for theories of particulars can be in danger of absurd inexistence and that graphical structuralism for theories of properties faces a similar danger, and a further danger of absurd cessation of change. To meet his challenge has required a sharper formulation of graphical 
structuralism and investigation of specific structural features corresponding to relevant metaphysical principles. The defence has revealed some important constraints. Particularist graphical structuralism is committed to four-dimensionalism and a finer level of structure than physics regards as fundamental, possibly finer than physics can treat as fundamental - this last being, perhaps, a defect. Property graphical structuralism is probably incompatible with the conjunction of immanence and presentism and requires the possibility of quiescent instantiation of potencies - which last might, also, be a defect. Finally, the Snowflake Lemma: that all finite uniquely manifestible connected potency graphs are 'snowflakes', is a surprising and perhaps important fact about the necessary structure of the fundamental level of properties under dispositional essentialism.

\section{Appendix}

A potency graph is a graph whose structure could be that had by a graph whose nodes are potencies and whose arrows link potencies to manifestation conditions. A manifestible graph has at least one arrow leaving each node. A uniquely manifestible graph has exactly one arrow leaving each node. A full walk starts at a node and keeps following arrows so long as it is able to. A manifestation regress from a potency regresses by iteratively tracing back from each potency already reached to all the potencies for which it is a

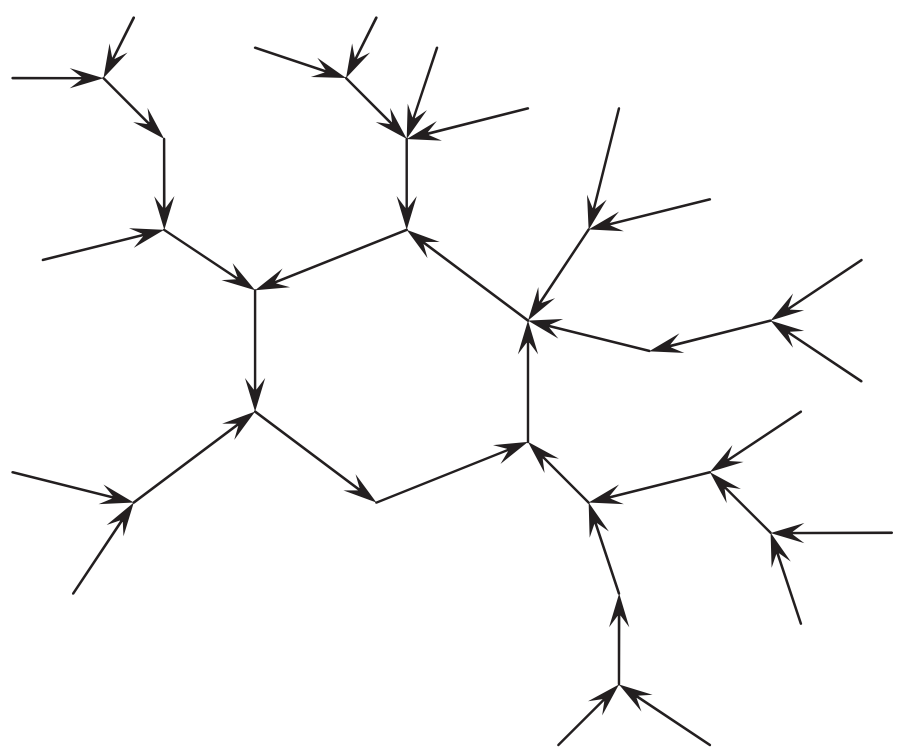

Figure A1. Snowflake. 
manifestation condition. A converging tree is one for which any full walk starting at any node ends at the same node, which is the final node of the tree. A snowflake is a graph composed of a one-way polygon each of whose nodes may also be the final node of a converging tree that shares no arrows with the polygon.

Lemma 2: The smallest potency graphs have 3 nodes.

Theorem 1: For any $k \geq 3$, there is a potency graph with $k$ nodes such that for some node, there is a manifestation regress from that node that reaches all potencies.

Theorem 2: There is a connected potency graph such that there is no node from which a manifestation regress reaches all potencies.

Theorem 3: There is a connected potency graph for which a manifestation regress leaves a subgraph that is a potency graph untouched.

Snowflake Lemma: All finite uniquely manifestible connected potency graphs are snowflakes.

Uniquely Manifesting Potencies Regress Theorem: For all uniquely manifestible connected potency graphs there is node from which a regress will reach all potencies.

Proofs available from the author.

\author{
University of Cardiff \\ Cardiff CF10 3EU \\ shackeln@cardiff.ac.uk \\ Oxford Uehiro Centre for Practical Ethics \\ University of Oxford \\ Oxford OX1 1PT \\ nicholas.shackel@philosophy.ox.ac.uk
}

\title{
References
}

Armstrong, D. M. 2008. Universals as attributes. In Metaphysics: Contemporary Readings, 2nd edn, ed. M. J. Loux, London; New York: Routledge.

Bird, A. 2007. Nature's Metaphysics: Laws and Properties. Oxford: Oxford University Press.

Dipert, R. R. 1997. The mathematical structure of the world: the world as graph. The Journal of Philosophy 94: 329-58.

Oderberg, D. S. 2011. The world is not an asymmetric graph. Analysis 71: 3-10. 